\title{
13. ON THE MOTION OF SHORT-PERIOD COMETS IN THE NEIGHBOURHOOD OF JUPITER
}

\author{
V. M. CHEPUROVA \\ Sternberg Astronomical Institute, Moscow, U.S.S.R.
}

\begin{abstract}
An intermediate orbit is developed for studying the hyperbolic jovicentric motion of a comet in the vicinity of Jupiter, where the effect of Jupiter's oblateness may become significant. A comparison is made with some of the results obtained by other investigators on the motions of specific periodic comets.
\end{abstract}

An intermediate orbit has been constructed (Chepurova, 1970) on the basis of the solution, advanced by Aksenov et al. $(1961,1963)$, of the generalized problem of two fixed centres. If the force function

$$
U=\frac{f m}{2}\left(\frac{1}{r_{1}}+\frac{1}{r_{2}}\right)
$$

is written in the form of the series

$$
U=\frac{f m}{r}\left[1+\sum_{k=1}^{\infty} \frac{\gamma_{2 k}}{r^{2 k}} P_{2 k}\left(\frac{z}{r}\right)\right]
$$

where $f$ is the gravitational constant, $m$ the mass of the central body, and $r=\left(x^{2}+y^{2}+z^{2}\right)^{1 / 2}$ the radius vector of a moving, massless particle; $r_{1}=\left[x^{2}+y^{2}+\right.$ $\left.(z-c i)^{2}\right]^{1 / 2}$ and $r_{2}=\left[x^{2}+y^{2}+(z+c i)^{2}\right]^{1 / 2}$, where $i=\sqrt{-1}$ and $c$ is the constant of the generalized two fixed centres problem; $P_{2 k}(z / r)$ are Legendre polynomials, and $\gamma_{2 k}=(-1)^{k}\left(c^{2 k} / 2\right)$. Specifically,

$$
\gamma_{2}=-J_{2} R_{0}^{2}
$$

where $J_{2}$ is the second harmonic of the potential of an axially symmetrical body, and $R_{0}$ is the equatorial radius of the body. As is well known, the potential of the axially symmetrical body has the form

$$
V=\frac{f m}{r}\left[1+\sum_{k=2}^{\infty} J_{k}\left(\frac{R_{0}}{r}\right)^{k} P_{k}\left(\frac{z}{r}\right)\right],
$$

where the zonal harmonics $J_{k}$ describe the gravitational field of the body (in the case of the planets of the solar system $J_{2}$ is much larger than all the other terms of the series). Then $U$ approximates $V$ sufficiently well. Comparison of calculations by the formulae of the intermediate orbit with the results of numerical integration of the differential equations of the motion

$$
\ddot{x}=\frac{\partial U}{\partial x}, \quad \ddot{y}=\frac{\partial U}{\partial y}, \quad \ddot{z}=\frac{\partial U}{\partial z}
$$

has shown that the intermediate orbit, which is the solution of Equations (3) and 
therefore involves the second harmonic of the potential of the central body, approximates the motion of the massless point in the gravitational field of the oblate body with axial symmetry much better than a Keplerian hyperbola.

The rectangular coordinates of a point moving along the intermediate orbit are expressed by the formulae

$$
\begin{aligned}
& x=\left(\zeta^{2}+c^{2}\right)^{1 / 2}(-\sin \varphi \sin \Omega+\cos \varphi \cos \Omega \cos i) \\
& y=\left(\zeta^{2}+c^{2}\right)^{1 / 2}(\sin \varphi \cos \Omega+\cos \varphi \sin \Omega \cos i) \\
& z=\zeta s \cos \varphi,
\end{aligned}
$$

where

$$
\begin{aligned}
\zeta & =a(1-e \bar{e}) \frac{1+\kappa \cos \psi}{1+\bar{e} \cos \psi} \\
\varphi & =\psi(1+\nu)+\bar{\omega}+\omega(\psi) \\
\Omega & =\Omega_{0}+\zeta(\psi),
\end{aligned}
$$

and the variable $\psi$ is connected with the time $t$ by the equations

$$
\begin{aligned}
& M=\bar{M}_{0}+\bar{n} \Delta t=e^{*} \tan E-\log \tan \left(\frac{E}{2}+\frac{\pi}{4}\right)+f_{1}(\psi) \\
& E=2 \arctan n^{\prime} \tan \frac{\psi}{2} .
\end{aligned}
$$

Here $a, e, s, \bar{\omega}, \Omega_{0}, \bar{M}_{0}$ and $\bar{n}$ are constants that become the Keplerian elements (or quantities connected with them) when $c=0$ and so may be called the elements of the intermediate orbit; $\omega(\psi), \zeta(\psi)$ and $f_{1}(\psi)$ are trigonometric series in $\psi$ with coefficients that depend on the quantities $\bar{e}, \kappa, \nu, e^{*}$ and $n^{\prime}$, as well as on the elements $a$, $e$ and $s$ of the intermediate orbit.

It is known that short-period comets of the Jupiter family may occasionally approach Jupiter so closely that the planet's oblateness, which usually influences them very little, begins to affect their motions. When investigating such very close approaches to a major planet it is usual to apply a planetocentric method for the calculation of the perturbations. After familiarizing herself with the work of Kazimirchak-Polonskaya (1961) the author had the idea of taking the first approximation to the motion in such cases to be the above-mentioned intermediate orbit, instead of a Keplerian hyperbola. This is the first attempt to construct this kind of analytical theory for the investigation of cometary motion, and thus for the time being we have not taken into account any other perturbations.

Cometary coordinates obtained from the formulae of the intermediate orbit have been compared with those given earlier by other investigators, notably Dubyago's (1950) investigation of P/Brooks 2 and Kamieński's (1949, 1956) investigation of $\mathrm{P} /$ Wolf over several close approaches to Jupiter. Some of the results are given in the Tables I, II and III, the coordinates being referred to Jupiter's equator and measured in AU. The tables show that the results are satisfactory enough, but in the future it will be necessary to take into account the perturbations by the Sun and the other planets. 
TABLE I

Coordinates of $\mathrm{P} /$ Brooks 2 at $t=1886$

November 3.0

\begin{tabular}{lll}
\hline & Dubyago & Intermediate orbit \\
\hline$x$ & -0.081157 & -0.082498 \\
$y$ & +0.277657 & +0.277604 \\
$z$ & +0.088909 & +0.095393 \\
\hline
\end{tabular}

The comet approached Jupiter on 1886 July 20.7657 to a distance of 0.0009636 AU.

TABLE II

Coordinates of $\mathrm{P} /$ Wolf at $t=1757$ February 16.0

\begin{tabular}{lll}
\hline & Kamieński & Intermediate orbit \\
\hline$x$ & -0.1403911 & -0.1434908 \\
$y$ & +0.1052629 & +0.1047257 \\
$z$ & +0.0932434 & +0.0921376 \\
\hline
\end{tabular}

The comet approached Jupiter on 1757 January 0.2 to a distance of $0.0758 \mathrm{AU}$.

TABLE III

Coordinates of $\mathrm{P} / \mathrm{Wolf}$ at $t=1875$ June 24.0

\begin{tabular}{lll}
\hline & Kamieński & Intermediate orbit \\
\hline$x$ & +0.0643464 & +0.0643479 \\
$y$ & +0.0119418 & +0.0119423 \\
$z$ & +0.1156719 & +0.1156721 \\
\hline
\end{tabular}

The comet approached Jupiter on 1875 June 8.7 to a distance of $0.1180 \mathrm{AU}$.

\section{References}

Aksenov, E. P., Grebenikov, E. A., and Demin, V. G.: 1961, Iskusstv. Sputniki Zemli No. 8, 64.

Aksenov, E. P., Grebenikov, E. A., and Demin, V. G.: 1963, Astron. Zh. 40, 363.

Chepurova, V. M.: 1970, Byull. Inst. Teor. Astron. 12, 216.

Dubyago, A. D.: 1950, Trudy Astron. Obs. Kazan No. 31.

Kamieński, M.: 1949, Bull. Acad. Polon. Sci. Lettres Ser. A 61.

Kamieński, M.: 1956, Acta Astron. 6, 153.

Kazimirchak-Polonskaya, E. I.: 1961, Trudy Inst. Teor. Astron. 7. 


\section{Discussion}

D. V. Zagrebin: Why have you done the expansion in Legendre polynomials, which are divergent - as was shown by Lyapunov?

$V . M$. Chepurova: I have done it in the traditional way. I am going to try other expansions in my future work.

G. N. Duboshin: The expansion of the potential gravitational field in Legendre polynomials is convergent, as was shown in the classical theory of gravitation, and it was quite appropriate for Chepurova to apply this expansion - just as it has been applied in all the papers on the motions of artificial satellites. Nevertheless it is possible that expansion in Lamé functions will be more rapidly convergent. But we lack now the appropriate mathematical operators to apply Lamé expansions directly. 